Philosophie ANTIQUE

\section{Philosophie antique}

Problèmes, Renaissances, Usages

$21 \mid 2021$

Les éléments

\title{
Étienne HELMER (dir.), Mendiants et mendicité en Grèce ancienne
}

Paris, Classiques Garnier, 2020 (Kaïnon - Anthropologie de la pensée ancienne, 13), ISBN : 978-2-406-09005-2

Donatella Izzo

\section{(2) OpenEdition}

Journals

Édition électronique

URL : https://journals.openedition.org/philosant/4245

DOI : $10.4000 /$ philosant.4245

ISSN : 2648-2789

Éditeur

Éditions Vrin

\section{Édition imprimée}

Date de publication : 15 décembre 2021

Pagination : 304-306

ISBN : 978-2-7116-3036-3

ISSN : 1634-4561

\section{Référence électronique}

Donatella Izzo, «Étienne heLmer (dir.), Mendiants et mendicité en Grèce ancienne », Philosophie antique [En ligne], 21 | 2021, mis en ligne le 12 mai 2021, consulté le 07 décembre 2022. URL : http:// journals.openedition.org/philosant/4245; DOI : https://doi.org/10.4000/philosant.4245

\section{(c) (i) (\$)}

Creative Commons - Attribution - Pas d'Utilisation Commerciale - Pas de Modification 4.0 International - CC BY-NC-ND 4.0

https://creativecommons.org/licenses/by-nc-nd/4.0/ 
Mendiants et mendicité en Grèce ancienne, sous la direction d'Étienne HeLmer, Paris, Classiques Garnier, 2020 (Kaïnon - Anthropologie de la pensée ancienne, 13), 309 p., ISBN : 978-2-406-09005-2.

Ce livre offre une vue d'ensemble sur la mendicité en traversant plusieurs disciplines (histoire, philosophie, littérature) et plusieurs genres littéraires (epos, tragédie, littérature philosophique) et en se déployant sur un arc chronologique qui s'étend de la Grèce archaïque jusqu'au monde judéo-chrétien. L'ouvrage se compose d'une présentation, rédigée par Étienne Helmer, et de onze contributions, organisées en trois volets : I. « Au centre et au bord. Espaces civiques et institutionnels du mendiant et de la mendicité en Grèce ancienne »; II. « Poétiques du mendiant. Mendiants et mendicité dans l'épopée, la tragédie et la littérature judéo-chrétienne »; III. « Sagesse folle ? La philosophie inquiétée par le mendiant et la mendicité ».

La première partie de l'ouvrage offre une vision générale de la condition du mendiant dans la société grecque et démontre que les sources anciennes, interrogées de façon intelligente, portent les traces d'une relative intégration. Lucia Cecchet («Intégrée, isolée, maudite. Formes, lieux et enjeux de la mendicité dans le monde grec ») montre que, malgré le fait que la morale traditionnelle assimilait la mendicité à une sorte de paresse et la considérait donc comme une menace potentielle pour la société, les Grecs étaient conscients que, souvent, il ne s'agissait pas d'une condition choisie mais de la conséquence d'un mauvais coup du sort. Selon cette perspective, les mendiants, victimes de la $\tau u ́ \chi \eta$, conservaient le droit à la pitié. Aida Fernández Pietro (« Le divin et la mendicité en Grèce ancienne ») analyse la problématique du point de vue de la religion et du rite : les sources suggèrent la présence d'un rapport plus ou moins direct entre certaines divinités et les mendiants. Maxime Chapuis (« Le va-nu-

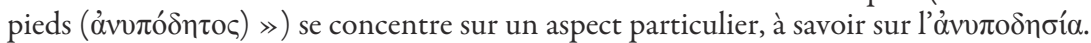
$\mathrm{Du}$ point de vue socio-économique et anthropologique, cet élément est négativement connoté : le «va-nu-pieds » se présente comme un individu qui refuse les produits de la technique, qui choisit la nature, conçue comme un état primitif, contre la culture. Il est donc un personnage anti-prométhéen et apolitique. Cependant, dans une perspective philosophique, et notamment à partir de la réflexion socratique, cet élément assume une connotation positive : renoncer aux chaussures est un signe de renonciation au superflu et donc d'autarcie. L'analyse de Chapuis anticipe ainsi par plusieurs points de vue celle développée dans la troisième partie.

La tragédie et la littérature judéo-chrétienne sont au cœur de la deuxième partie. Amandine Gouttefarde («Expier sur les routes. Exil, mendicité et souillure dans la littérature grecque ») étudie le rapport entre exilé et mendiant à travers l'analyse de passages tirés d'Homère, d'Hérodote, des tragédiens, d'Isocrate. La chercheuse montre que l'exilé, qui devient souvent un $\pi \tau \omega \chi \chi_{\varsigma}$, est parfois porteur d'une souillure, mais qu'il peut vivre une expérience de purification grâce à l'exil et être donc admis de nouveau au sein de la communauté. Nathalie Assan Libé («Un mendiant pour les uns, un allié pour les autres. Mendicité, supplication et hospitalité dans l'OEdipe à Colone de Sophocle ») se concentre surtout sur le personnage d'OEdipe. Son analyse rhétorique montre que les différents personnages de l'CEdipe à Colone de Sophocle se servent d'allusions à la misère d'Antigone et d'OEdipe pour servir leurs buts rhétoriques. En particulier, dans la perspective de Thésée, OEdipe n'est pas un mendiant : c'est plutôt un hôte. Michel Briand («Ulysse mendiant à Ithaque. Kinesthésie, jeux d'empathie et d'identité ») s'intéresse à la figure de l'Ulysse mendiant, et en particulier à son style kinésique : ses gestes, ses mouvements et ses postures en rapport avec ses paroles et son regard. Briand analyse trois traits kinésiques liés à la mendicité dans l'Odyssée : l'errance, la prostration et la 
quête. Cette approche met en évidence le fait que les autres personnages se caractérisent par la façon dont ils réagissent à la présence du nouveau mendiant : Eumée, Télémaque, la nourrice Euryclée, Pénélope respectent les principes de l'hospitalité et de l'accueil, à la différence des prétendants, et notamment d'Antinoos, de la servante Mélanthô et d'Iros. La contribution de Sandrine Coin-Longeray (« Messager des temps nouveaux. Le mendiant dans la littérature judéo-hellénique et des origines du christianisme ») prend appui sur le présupposé voulant que le mendiant soit méprisé dans la littérature païenne et soit présent dans la littérature patristique du $\mathrm{IV}^{\mathrm{e}}$ siècle comme une figure sacrée. La chercheuse se concentre donc sur le moment intermédiaire entre ces deux phases, celui de la littérature judéo-hellénique et du christianisme primitif. Malgré la présence d'éléments importants comme l'obligation morale de la charité et l'interdiction de profiter de la faiblesse du mendiant, la solidarité envers ce dernier se révèle un concept tardif. D'ailleurs, dans la littérature chrétienne, le partage du repas remplace le devoir de la charité et l'obligation à une participation émotionnelle réintègre dans une certaine mesure le mendiant dans la société.

La troisième partie analyse la problématique du point de vue philosophique. LouisAndré Dorion (« De quoi vit Socrate ? Le refus de la mendicité et la tentation des cadeaux ») se questionne sur les moyens de subsistance de Socrate, tout en soulignant que le problème du Socrate historique demeure insoluble et qu'il est plutôt question de réfléchir à la représentation du philosophe dans les différentes sources. Il se concentre notamment sur les témoignages de Platon, Xénophon et sur les sources tardives. Les trois derniers articles sont consacrés au cynisme, philosophie déjà évoquée dans le titre de la dernière partie, l'expression « sagesse folle » renvoyant à la célèbre définition de Diogène comme Socrate devenu fou attribuée à Platon (cette définition est discutée à la p. 242). Le grand espace consacré au Cynisme était attendu dans un ouvrage dédié à la mendicité, car le cynisme en propose un renversement sous le signe du paradoxe : mendier dans sa double signification d'être misérable et de quémander n'est pas seulement considéré comme une attitude acceptable, mais fait partie du modus vivendi du philosophe. Isabelle Chouinard (《Le sage peut-il mendier ? ») discute de l'attitude d'Aristippe, des Épicuriens et des Stoïciens vis-à-vis de la mendicité cynique : si le choix du dépouillement total est parfois accepté par d'autres philosophes, les cyniques sont les seuls à faire la quête. Étienne Helmer («L'autarcie à l'épreuve de la mendicité cynique ») se focalise sur la contradiction seulement apparente entre autarcie et mendicité : la mendicité cynique est conceptuellement liée à la désappropriation individuelle (tout est à tous) et respecte, contre toute attente, le principe de réciprocité. Olimar Flores-Júnior (« Lire et écrire dans les marges. Activité littéraire et mendicité chez les cyniques grecs ») réfléchit à un autre paradoxe apparent lié aux Cyniques, à savoir celui de l'écriture. L'article remet en question le refus de la paideia de la part des Cyniques et démontre que, malgré leur misère, la littérature fait partie des $\alpha \nu \alpha \gamma \kappa \alpha i ̃ \alpha$ du philosophe cynique.

Unebibliographie très riche, un indexnominum, un indexrerum, un indexlocorumetles résumés de chaque contribution complètent le volume. Il est plutôt surprenant que Télès, une source parmi les plus importantes pour le Cynisme et citée très souvent, notamment dans la troisième partie, soit absent de l'index nominum aussi bien que de l'index locorum.

En conclusion, cet ouvrage montre bien que le statut du mendiant était très ambigu, « à la fois dehors et dedans », selon la définition d'Étienne Helmer (p. 8) et que finalement il n'était pas « aussi marginal qu'il le semble ». La multiplicité des sources interrogées et les différentes approches permettent de réfléchir à la dialectique entre mendicité et d'autres notions anthropologiques, telles que le parasitisme, la pauvreté, l'exil, l'hospitalité, l'autarcie. On peut regretter cependant que la comédie, citée ici et là 
plutôt rapidement, n'ait pas bénéficié de plus d'attention. Par exemple, il aurait pu être intéressant de s'interroger sur la raison pour laquelle la misère était souvent présente sur la scène comique. En outre, la comédie aurait permis d'explorer davantage le rapport entre la figure du mendiant et celle du parasite (Lucia Cecchet évoque rapidement ces questions aux p. 24 et 28). Le Socrate d'Aristophane aurait peut-être mérité une plus grande attention dans l'article de Louis-André Dorion. Léonidas de Tarente aurait aussi mérité d'être mentionné dans le volume, en considérant ses nombreuses allusions à la misère et les influences très débattues du cynisme sur sa production.

Ces petites remarques n'ôtent rien à la valeur de cet ouvrage. Il s'agit non seulement d'un volume riche d'informations et de résultats originaux, mais aussi de l'un des rares volumes entièrement dédiés à cette problématique (je signale la récente publication de l'ouvrage de Pietro Giammellaro, Il mendicante nella Grecia antica. Teorie e modelli, Brescia, 2019), deux éléments qui en font une référence incontournable pour les chercheurs qui s'occupent de misère et de mendicité dans l'Antiquité, mais aussi une lecture intéressante et plaisante pour les non-spécialistes.

Donatella Izzo

CERAM - Sorbonne Nouvelle. Collège Stanislas, Paris

Sophie Klimis, Le penser en travail. Castoriadis et le labyrinthe de la création humaine. [1] Polis. De la société capitaliste à la société des Athéniens, Presses Universitaires de Paris Nanterre, 2020 (Au détour des anciens, 2), 456 p., ISBN : 978-2-84016-375-6.

Avec le premier volume d'une future trilogie consacrée respectivement aux trois principaux « carrefours » du « penser »de Cornelius Castoriadis (C. C.), la polis, la psychè et le logos, Sophie Klimis (S. K.) nous propose en réalité une enquête sur notre modernité à travers la notion castoriadienne de « figure du pensable (eidos) socialhistorique ». Selon elle, l'un des mérites de C. C. est d'avoir saisi que notre modernité est complexe, articulant à une « modernité des Modernes », le capitalisme, une « modernité des Anciens » qui se confond avec un projet politique d'autonomie (p. 352). Comme en atteste le sous-titre de l'ouvrage, véritable titre du volume consacré à la Polis, notre modernité se donne donc à lire à partir d'une relecture de Marx et de la démocratie athénienne des $\mathrm{V}^{\mathrm{e}}$ et $\mathrm{IV}^{\mathrm{e}}$ siècles. Non moins suggestif dans ce titre est le sens du mouvement, de Marx aux Anciens, qui illustre à la fois les phases de la pensée de C. C., mais aussi son appel à dépasser le capitalisme dans sa forme néo-libérale vers une rénovation du «germe » grec (p. 183). Pourtant, en abordant ainsi « l'axe grécocontemporain $\gg, S$. K., tout en souscrivant pleinement à la tentative de relance du projet politique d'autonomie initié par C. C., apporte une modification substantielle à ses analyses. Elle se propose ainsi de mettre en évidence ce qu'il n'a, selon elle, pas su ou pu voir pleinement, à savoir « que le projet de maîtrise rationnelle sur le tout et le projet d'autonomie sont les deux facettes complémentaires d'une seule et même signification imaginaire » (p. 123). C'est pourquoi, à côté des tentatives contemporaines inabouties de relance du projet d'autonomie au sein du capitalisme (mouvement ouvrier, p. 145-151, ou mouvement des places, p. 401-405), il faut également selon S. K. lire dans la démocratie grecque les germes du projet de maîtrise rationnelle constitutif du capitalisme (p. $228 ;$ p. 367-373).

La première partie de l'ouvrage revient sur la notion de « forme du pensable socialhistorique », qu'elle analyse à la fois comme concept et à travers deux illustrations 